\title{
A rare case of superior vena cava syndrome in a patient on VV-ECMO
}

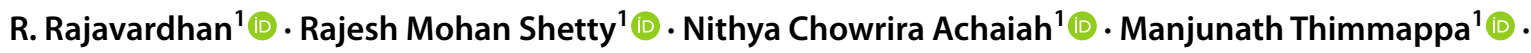 \\ Arun Veeram Reddy ${ }^{2}$
}

Received: 15 July 2021 / Revised: 14 October 2021 / Accepted: 18 October 2021 / Published online: 4 January 2022

(C) Indian Association of Cardiovascular-Thoracic Surgeons 2021

\begin{abstract}
Superior vena cava (SVC) syndrome is caused by either extrinsic compression of SVC or intrinsic lesions within SVC leading to obstruction of SVC and consequently swelling of the face, neck, and upper extremeities. Iatrogenic incidence is on the rise due to the use of long-term indwelling catheters. SVC syndrome in extracorporeal membrane oxygenation (ECMO) has been defined in neonatal and paediatric ECMO cases. Only one case of SVC syndrome in adult has been defined while using a double-lumen ECMO cannula. Our case describes a case of SVC syndrome in a case on veno-venous ECMO (VVECMO) with an internal jugular vein (IJV) return cannula which is unheard of. A high index of suspicion is required to diagnose SVC syndrome.
\end{abstract}

Keywords ECMO $\cdot$ COVID-19 $\cdot$ SVC syndrome $\cdot$ ARDS

\section{Introduction}

Superior vena cava syndrome is a spectrum of manifestations caused by either partial or complete compression of the superior vena cava (SVC). It is characterized by swelling of the upper part of the body. SVC is formed by the joining of the two brachiocephalic veins which drain the blood from the head, neck, and upper extremities. The most common cause of SVC obstruction is malignancy. SVC syndrome is usually associated with neonatal and paediatric extracorporeal membrane oxygenation (ECMO) patients. One case of SVC syndrome was recently reported in an adult patient on

R. Rajavardhan

rajavardhanr99@gmail.com

Rajesh Mohan Shetty

drrajeshshetty@gmail.com

Nithya Chowrira Achaiah

nithyaachaiah@gmail.com

Manjunath Thimmappa

manjut110@yahoo.co.in

Arun Veeram Reddy

arunodhaya1979@gmail.com

1 Critical Care Medicine, Manipal Hospital Whitefield, Bangalore, Karnataka, India

2 Cardiothoracic Surgery, Manipal Hospital Whitefield, Bangalore, Karnataka, India
ECMO with the right internal jugular vein (IJV) dual-limb cannula. In this case report, we describe a case of SVC syndrome associated with adult ECMO with IJV single-lumen cannula.

\section{Case report}

A 78-year-old male with coronavirus disease 2019 (COVID19) pneumonia with hypoxemic respiratory failure was admitted to our hospital. He was intubated and right-sided intercostal drainage was inserted in view of pneumothorax. He was prone ventilated because of refractory hypoxemia. On day 3, given persistent hypoxemia and hypercapnia, the decision to initiate veno-venous ECMO (VV-ECMO) was made (Maquet Cardiohelp HLS Set Advanced 7.0). Right femoral vein access drainage cannula of $27 \mathrm{Fr}$ and return right IJV cannula of 21-Fr dimensions were inserted and initiated on unfractionated heparin anticoagulation. Following the initiation of ECMO, flow issues were noted immediately. Echocardiography did not show any anatomical abnormalities and showed under filled ventricles indicating hypovolemia. Haemoglobin monitoring did not show any reduction ruling out occult bleeding. Computed tomography (CT) of the chest plain done before initiation of ECMO did not show any major anatomical abnormalities. The patient was resuscitated with intravenous fluids. Over the next few hours, 


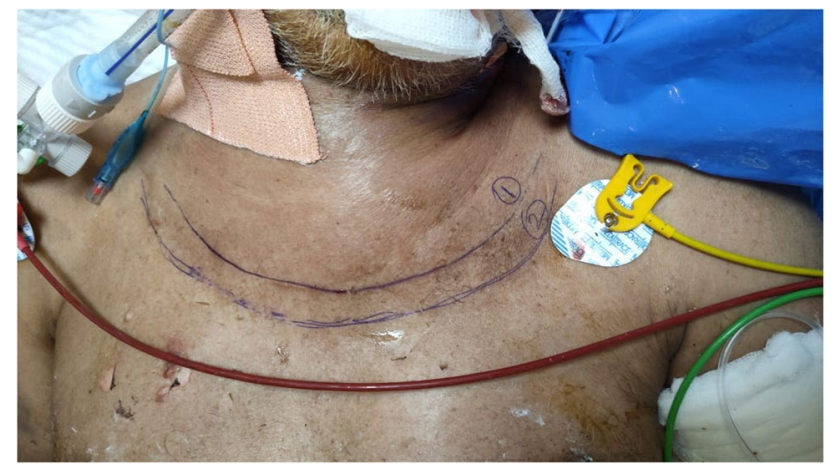

Fig. 1 Picture showing rapidly increasing neck swelling with lines marked 1 and 2 showing rapidly increasing neck swelling within few hours

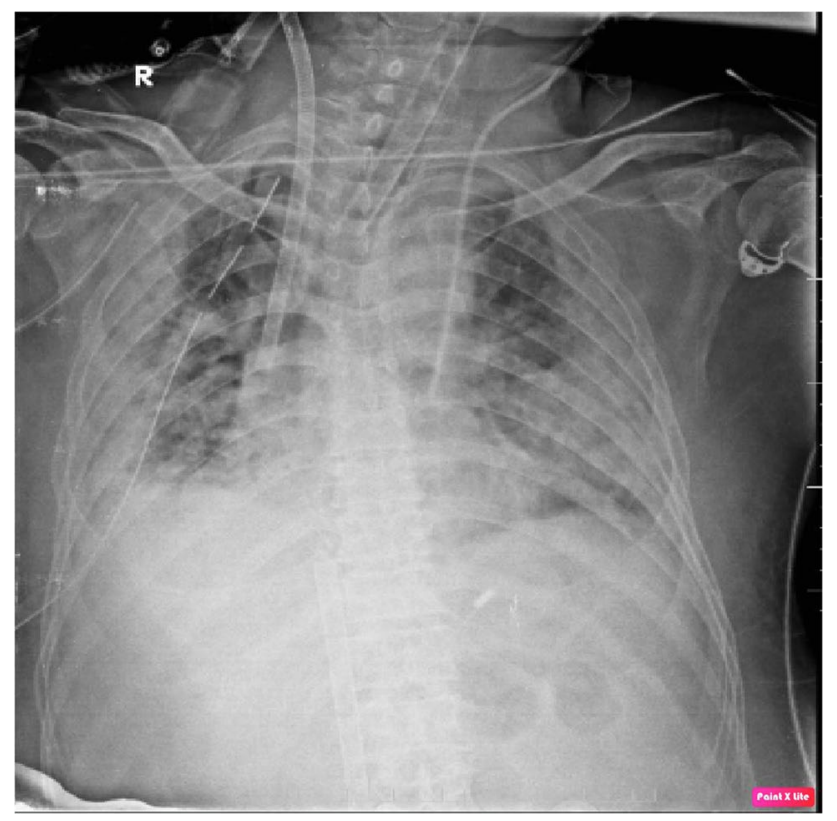

Fig. 2 X-ray showing left-sided persistent SVC with extracorporeal membrane oxygenation cannulas and right-sided intercostal drainage

the patient was noted to have repeated episodes of chattering requiring repeated fluid boluses. Vasopressors were initiated in view of the onset of shock. Neck swelling was noted which gradually increased in size (Fig. 1). Bleeding was suspected from the cannulation site. Ultrasound of the neck was done, which showed only soft tissue swelling and ruled out haematoma or thrombosis of the IJV. Over the next few hours, facial swelling and conjunctival oedema were noted. Chest X-ray post cannulation showed central vein cannulation in left-sided persistent SVC (Fig. 2). Therefore, it was decided to reposition the return cannula in the IJV from $15 \mathrm{~cm}$ to the skin entry site to $12 \mathrm{~cm}$ and the left-sided central vein cannula was removed for better drainage from the upper part of the body. Over the next $24 \mathrm{~h}$, flow issues settled with regression of the swelling from the face and the neck. Vasopressors were gradually tapered and stopped with the resolution of lactic acidosis.

The patient was on ECMO for a total of 14 days. There were no further episodes of SVC obstruction.

\section{Discussion}

SVC is a major blood vessel draining blood from the upper part of the body. It is a low-pressure system with a thin wall, which is easily compressible. The incidence of SVC syndrome in the patients ranges from 1 in 650 to 1 in 3100 . The most common cause of SVC syndrome is due to extrinsic compression. It is usually due to mediastinal malignancies which include bronchogenic carcinoma, non-Hodgkin's lymphoma followed by metastatic tumour [1]. Non-malignant causes account for about $20 \%$ of the cases. It includes mediastinal fibrosis, infections like tuberculosis, pericarditis, benign tumours, aortic aneurysm, and thrombosis [2, 3]. It is caused by thrombosis which causes intrinsic compression. SVC syndrome manifests as swelling of the upper part of the body with include the neck, face, lips, and upper limbs. It manifests as cough, breathlessness, stridor, chest pain, orthopnea [4].

SVC obstruction is usually chronic as collaterals get established which divert blood from the upper part of the body to the lower part. It includes an azygous venous system connecting azygous veins to intercostals, internal mammary venous system, and long thoracic venous system [5]. CT and magnetic resonance imaging (MRI) scans will aid in the diagnosis by identifying thrombus, site of occlusion, and establishing the presence of collaterals. In the case of ECMO cannulation, it occurs in an acute situation and requires a very high index of clinical suspicion as imaging will be of little help.

SVC syndrome has been described in the paediatric population undergoing ECMO [6]. It is very rare in adults given the large caliber of the vessel. There have been case reports of it during intraoperative cannulation for cardiopulmonary bypass [7]. There is one case report of SVC syndrome during VV-ECMO in an adult. In this case, the patient was cannulated with a double-lumen IJV cannula [8]. This patient had a background history of chronic kidney disease requiring haemodialysis indicating the possibility of central narrowing due to a previous catheter. One more case of SVC syndrome has been described in the setting of veno-arterial (VA)-ECMO due to inadequate drainage from SVC and was treated with addition of SVC drainage cannula [9].

In our case, a high index of suspicion based on the clinical parameters and echocardiography, chest X-ray, and ultrasound of the neck helped us in ruling out the other 
possible causes and narrowing it to SVC syndrome. The patient had a left-sided persistent SVC indicating abnormal anatomy, most likely associated with narrow SVC. The presence of the left-sided central vein cannula also might have worsened it.

Acute SVC syndrome is important to diagnose as it may cause immediate haemodynamic collapse due to decreased venous return. It may also cause blindness, nerve injury, cerebral oedema, and respiratory compromise. Therefore, it is very important to choose appropriate size cannulas and to rule out underlying anatomical abnormalities.

\section{Conclusion}

Our case shows that it requires a high index of suspicion to diagnose SVC syndrome in the setting of ECMO. Patients developing swelling in the upper part of the body, face, and neck should undergo an evaluation to rule out SVC syndrome. It can be prevented by carefully selecting the appropriate size cannula and positioning them under guidance.

Funding None.

\section{Declarations}

Consent for publication Written informed consent was acquired from the patient for publication of this report.

Ethical committee approval Ethical committee clearance was taken.
Conflict of interest The authors declare no competing interests.

\section{References}

1. Escalante CP. Causes and management of superior vena cava syndrome. Oncology (Williston Park). 1993;7:61-8.

2. Akoglu H, Yilmaz R, Peynircioglu B, et al. A rare complication of hemodialysis catheters: superior vena cava syndrome. Hemodial Int. 2007;11:385-91.

3. Rice TW, Rodriguez RM, Light RW. The superior vena cava syndrome: clinical characteristics and evolving etiology. Medicine (Baltimore). 2006;85:37-42.

4. Ahmann FR. A reassessment of the clinical implications of the superior vena caval syndrome. J Clin Oncol. 1984;2:961-969.

5. Mansour A, Saadeh SS, Abdel-Razeq N, Khozouz O, Abunasser M, Taqash A. Clinical course and complications of catheter and non-catheter-related upper extremity deep vein thrombosis in patients with cancer. Clin Appl Thromb Hemost. 2018;24:1234-40.

6. Zreik H, Bengur AR, Meliones JN, Hansell D, Li JS. Superior vena cava obstruction after extracorporeal membrane oxygenation. J Pediatr. 1995;127:314-6.

7. Kopanczyk R, Al-Qudsi OH, Ganapathi AM, Potere BR, Pagel PS. Superior vena cava syndrome during veno-venous extracorporeal membrane oxygenation for COVID-19. Perfusion. 2021;36:630-633.

8. Niazi AK, Reese AS, Minko P, O'Donoghue D, Ayad S. Superior vena cava syndrome and otorrhagia during cardiac surgery. Cureus. 2019;11:e4602.

9. Amin S, Chweich H, Reich J, Garpestad E. Thoracic central vein occlusion causing lung collapse in a patient on venoarterial extracorporeal membrane oxygenation. Chest. 2019;156:A120.

Publisher's note Springer Nature remains neutral with regard to jurisdictional claims in published maps and institutional affiliations. 